\title{
Diagnostic Accuracy of Multidetector Computed Tomography in Evaluation of Retroperitoneal Traumatic Injuries and Retroperitoneal Hemorrhage
}

\author{
HAYTHAM H. EL-SAID, M.D.*; MOHAMMED M. DAWOUD, M.D.*; MAGED M. RAGAB, M.D.** and \\ MUSTAFA E.E. EL-HAGER, M.Sc.*
}

The Departments of Radiodiagnosis* and Urology**, Faculty of Medicine, Tanta University

\begin{abstract}
Background: Abdominal trauma represents a main daycare activity in radiology.non surgical treatment has become the standard of care in hemodynamically stable abdominal trauma patients as a result of comprehensive assessment of injury by imaging.

MDCT is very sensitive method to detect traumatic injuries and determine the grading of this injuries on which the management will be performed.

Aim of Study: To assess diagnostic accuracy of multidetector CT in evaluation of retroperitoneal traumatic injuries and retroperitoneal hemorrhage.

Patients and Methods: Prospective study included 60 patients with abdominal trauma (40 male, 20 females), their age ranged from 7 years to 70 years (mean age 34.8 year). Ultrasonography and MDCT were performed for all patients.

Results: 30 patients (50\% of the total studied patients) had retroperitoneal injuries, 27 patients of them $(90 \%)$ were secondary to blunt abdominal trauma and 3 patients $(10 \%)$ suffered from stab trauma. Fifteen patients had renal injuries; 3 of them associated with adrenal injuries, 2 patients had bowel injuries, 5 patients had pancreatic injuries, 3 patients had vascular injuries and 5 patients had retroperitoneal hematoma related to other injuries; 3 of them had psoas muscle injury and the other 2 patients had urinary bladder rupture.

Conclusion: MDCT proved to be accurate and sensitive to evaluate retroperitoneal traumatic injuries and determine the grading and severity of these injuries on which the management will be performed. In addition MDCT proved to be highly sensitive in detection of active hemorrhage which is a life threatening condition.
\end{abstract}

Key Words: Retroperitineal - Multidetector CT - Trauma.

Correspondence to: Dr. Haytham H. El-Said, The Department of Radiodiagnosis, Faculty of Medicine, Tanta University

\section{Introduction}

RETROPERITONEAL injuries occur in a low percentage as a result of abdominal and pelvic trauma, however, its occurrence is very challenging and poses a significant risk to patients' life [1]

Recently, multidetector computed tomography considered a valuable change along the way of CT development. This new technology has a great increase in acquisition speed, improved spatial resolution, intravenously administered contrast material bolus timing and reduced motion artifacts. Multidetector CT had an overall sensitivity of $94 \%$, specificity of $100 \%$ and accuracy of $97 \%$ in the evaluation of high-energy trauma injuries $[2,3]$.

Renal injuries occurs up to be reckoned with among $8-10 \%$ of patients admitted to an emergency department because of abdominal trauma. In 80$95 \%$ of the cases renal injuries are due to blunt trauma, whereas penetrating traumas cause the remaining 5-20\%. Renal trauma may occur isolated or in association with other visceral injuries [4].

The most widely used classification system for renal injuries is the American Association for the Surgery of Trauma (AAST) grading system which

\footnotetext{
Abbreviations:

3D : Three Dimensions.

AAST : American Association for the Surgery of Trauma.

CT : Computed Tomography.

CTA : Computed Tomography Angiography.

FAST : Focused Assessment with Sonography for Trauma

MDCT : Multi Detector Computed Tomography.

MIP : Maximum Intensity Projection.

MPR : Multiplanar Reformat.

PCS : Pelvicalyceal System.

US : Ultrasonography.
} 
depend on surgical findings (the standard for renal injury staging). Renal trauma is divided into five categories (grades I-V), according to the severity and depth of injury and involvement of the vasculature or collecting system [4].

Pancreatic and duodenal injuries are rare especially with blunt abdominal trauma representing only $2 \%$ of all blunt abdominal trauma. However, it is very important for radiologists to be aware of these injuries to avoid underestimation as it has a poor prognosis and decreases survival with reported mortalities of up to $30 \%$ in patients with blunt pancreatic trauma and up to $25 \%$ in patients with duodenal injuries $[\mathbf{5 , 6 ]}$.

Vascular injuries can occur spontaneously (nontraumatic) or as a result of direct traumatic injury to the vessel. Theses injuries can also be divided according to the type of involved vessels into arterial injury and venous injury. Vascular injuries following blunt (non-penetrating) trauma is a rare but life-threatening. Recent autopsy series have shown the incidence of abdominal aortic injuries ranging from 12 to $15 \%[7,8]$.

The rarity of aortic traumatic injury is attributed to its retroperitoneal location which makes it protected by the abdominal wall anteriorly, the visceral organs posteriorly, the vertebrae and the thick paravertebral musculature laterally [9].

The aim of this study to assess diagnostic accuracy of MDCT in evaluation of retroperitoneal traumatic injuries and highlight the problems in diagnosis and treatment to facilitate the surgeons to make decision that early diagnosis and correct treatment is important.

\section{Patients and Methods}

\section{Patients:}

Prospective study was conducted on 60 patients (40 male and 20 female), aged from 7 to 70 years old,with abdominal trauma throughout the period from December 2016 to November 2017 in the Radiology Department Tanta University Hospital. Informed consent were taken from all patients, they were informed about the procedure and its expected complications. This study was approved by the ethics committee of our institution.

Inclusion criteria were positive Focused Assessment with Sonography in Trauma (FAST) examination, negative FAST examination and hypotension (systolic blood pressure $<90 \mathrm{mmHg}$ ) and all penetrating injuries to the abdomen, flank or lower thorax. Both sexes will be included, no age predi- lection. Exclusion criteria were severely shocked patients who could not be transported to CT Unit, patients with contrast allergy and pregnancy.

The aetiology of retroperitoneal traumatic injuries were classified as the following; 27 (90\%) patients were secondary to blunt abdominal trauma; 11 of them due to motor vehicle accidents, 8 of them due to motorcycle accidents, 3 of them fell from height, 2 of them injured in sport activity and 3 of them injured in battle. Three (10\%) patients suffered from stab injuries. The above findings are listed in (Table 1).

Table (1): Aetiology of trauma among the patients included in our study with retroperitoneal injuries.

\begin{tabular}{lcl}
\hline Aetiology of trauma & No. of patients & \multicolumn{1}{c}{$\%$} \\
\hline Motor vehicle accidents & 11 & 36.6667 \\
Motorcycle accidents & 8 & 26.6667 \\
Falling from height & 3 & 10 \\
Sport injury & 2 & 6.6667 \\
Battle & 3 & 10 \\
Stab injury & 3 & 10 \\
\hline Total & 30 & 100 \\
\hline
\end{tabular}

\section{Methodology:}

Full history taking focused on present complaint e.g. mode of injury, abdominal pain, hematuria, etc. Clinical examination including general, local abdominal and pelvic examinations. Laboratory investigations including renal function and coagulation profile to assure proper renal function and exclude coagulation defects as cause of the hematuria.

Ultrasonography was done for all patients $(n=60)$ for assessment of blood clots in renal calyces, pelvis and/or urinary bladder or perinephric hematoma, intra abdominal free fluid, vascular lesions as aneurysm, retroperitoneal hematoma and peripancreatic fluid collection.

The patients were evaluated with MDCT and various injuries were graded according to American Association for the Surgery of Trauma (ASST). All patients were examined using a 128-slice CT (GE Optima model 660), non ionic contrast media (Omnipaque 300 mgI $/ \mathrm{ml}$, GE Healthcare, Cork, Ireland) was used. All patients were asked to fast for 4-6 hours before the procedure. The previous laboratory and radiological investigations were reviewed. Oral contrast is not routinely given.

An initial non-enhanced study is helpful in detecting acute bleeding or intra-parenchymal hematoma that may appear isoattenuating relative 
to the normal parenchyma at post-contrast $\mathrm{CT}$, then scanning at arterial phase (bolus tracking), venous phase (70-80 seconds delay) and delayed phase, CT angiography was done for suspected vascular injury. Post processing coronal and sagittal reformatted images were routinely obtained using multiplanar reconstruction technique from volumetric and isotropic axial CT data. The maximum intensity projection and $3 \mathrm{D}$ volume reconstruction could be obtained as needed.

\section{Results}

Prospective study included 60 patients with abdominal trauma, $40(66.7 \%)$ of them were males and $20(33.3 \%)$ of them were females, their ages ranged from 7 years to 70 years (mean age 34.85 year).

The current study consists of patients in which the traumatic retroperitoneal injuries was confirmed with a final diagnosis by MDCT and the patients did not have retroperitoneal injuries were excluded from this study.

Thirty patients had retroperitoneal injuries proved by ultrasonography and multi-detector computed tomography and the other 30 patients did not have retroperitoneal injuries; 23 patients had hepatic and splenic injuries and 7 patients had only hemoperitoneum.

Thirty patients had retroperitoneal injuries, 19 $(63 \%)$ of them were males and $11(37 \%)$ of them were females, their age distribution ranged from 9 years to 70 years (mean age 39.5 year). Clinically the 30 patients with retroperitoneal injuries were subdivided into; 26 patients $(86.67 \%)$ had abdominal pain, 11 patients $(36.67 \%)$ had haematuria and 8 patients $(26.67 \%)$ had deterioration of vital signs.

By MDCT, fifteen patients had renal injuries three of them associated with adrenal injuries, 2 patients had bowel injuries, 5 patients had pancreatic injuries, 3 patients had vascular injuries and 5 patients had retroperitoneal hematoma related to other injuries; 3 of them had psoas muscle injury, 2 patients had urinary bladder rupture. The five patient patients with retroperitoneal hematoma had pelvic fracture and one of them had vertebral fracture. These finding listed in (Table 2).

Sixteen patients had retroperitoneal injuries associated with other intraperitoneal injuries; 6 patients had splenic injury, 6 patients had hepatic injury and 4 patient had hepatic and splenic injuries.

Among 15 patients proved by MDCT had traumatic renal injuries; 2 patients had renal contusions,
2 patients had subcapsular haematoma, 4 patients had perinephric haematoma, 1 patient had renal parenchymal deep laceration not reaching the pelvicalyceal system, 2 patients had renal lacerations with contrast extravasation (urinoma), 4 patients had segmental renal infarction, 1 patient had shattered kidney and 3 patients had main vascular pedicle avulsion and non perfused kidney. The above finding shown in (Table 3 ).

According to the American Association of Surgery of Trauma (AAST), these patients were classified as followed: 3 patients with grade I renal trauma, 2 patients with grade II renal trauma, 1 patient with grade III renal trauma, 5 patients with grade IV renal trauma and 4 patients with grade $\mathrm{V}$ renal trauma.

Five patients had pancreatic injuries proved by MDCT; one patient had superficial linear laceration, 2 patients had major contusion less than one half of pancreatic depth, one patient had deep pancreatic laceration more than one half of pancreatic depth and the last patient had transection through the full thickness of the pancreatic neck. According to the American Association of Surgery of Trauma (AAST); they were classified as followed: One patient with grade I pancreatic traumatic injury, one patient with grade II pancreatic traumatic injury, one patient with grade III pancreatic traumatic injury and the last patient with grade IV pancreatic transection.

Two patients had bowel injuries proved by MDCT, one of them had duodenal perforation at its descending segment (part II) with extraluminal air leak associated with left hepatic lobe multiple deep lacerations, the other patient had duodenal contusion with thickening of the duodenal wall in its transverse part (part III) with no extraluminal air.

Three patients had vascular injuries by MDCT, one patient had rupture abdominal aortic aneurysm forming central retroperitoneal hematoma (zone I) with post traumatic pseudoaneurysm formation, one patient had right common iliac artery injury forming pelvic hematoma (zone III) and the other patient had post traumatic mesenteric pseudoaneurysm (zone I).

Five patients had retroperitoneal hematoma related to other injuries, 3 patients had psoas muscle injury and the other 2 patients had urinary bladder rupture, the five patients had pelvic fracture and one of them had vertebral fracture. 
Twenty patients with retroperitoneal injuries managed conservatively and 10 patient managed operatively. The operated patients were correlated with the CT findings and most of the conservative

Table (2): Types of the retroperitoneal injuries among the studied patients.

\begin{tabular}{|c|c|c|c|c|c|}
\hline Types of injuries & Number of patients & $\%$ & MDCT findings & No. of cases & $\%$ \\
\hline Renal and adrenal injuries & 15 & 50 & Renal contusion & 2 & 13.333 \\
\hline Bowel injuries & 2 & 6.667 & Renal subapsular haematoma & 2 & 13.333 \\
\hline Pancreatic injuries & 5 & 16.667 & Perinephric haematoma & 4 & 26.667 \\
\hline & 3 & & Renal parenchymal laceration $>1 \mathrm{~cm}$ & 1 & 6.667 \\
\hline vascuiar injuries & 5 & 10 & Renal lacerations extending to the PCS & 2 & 6.667 \\
\hline Retroperitoneal hematoma & 5 & 16.667 & Renal segmental vascular injury & 4 & 26.667 \\
\hline Total & 30 & 100 & Renal main vascular pedicle avulsion & 3 & $\begin{array}{l}0.001 \\
20\end{array}$ \\
\hline
\end{tabular}
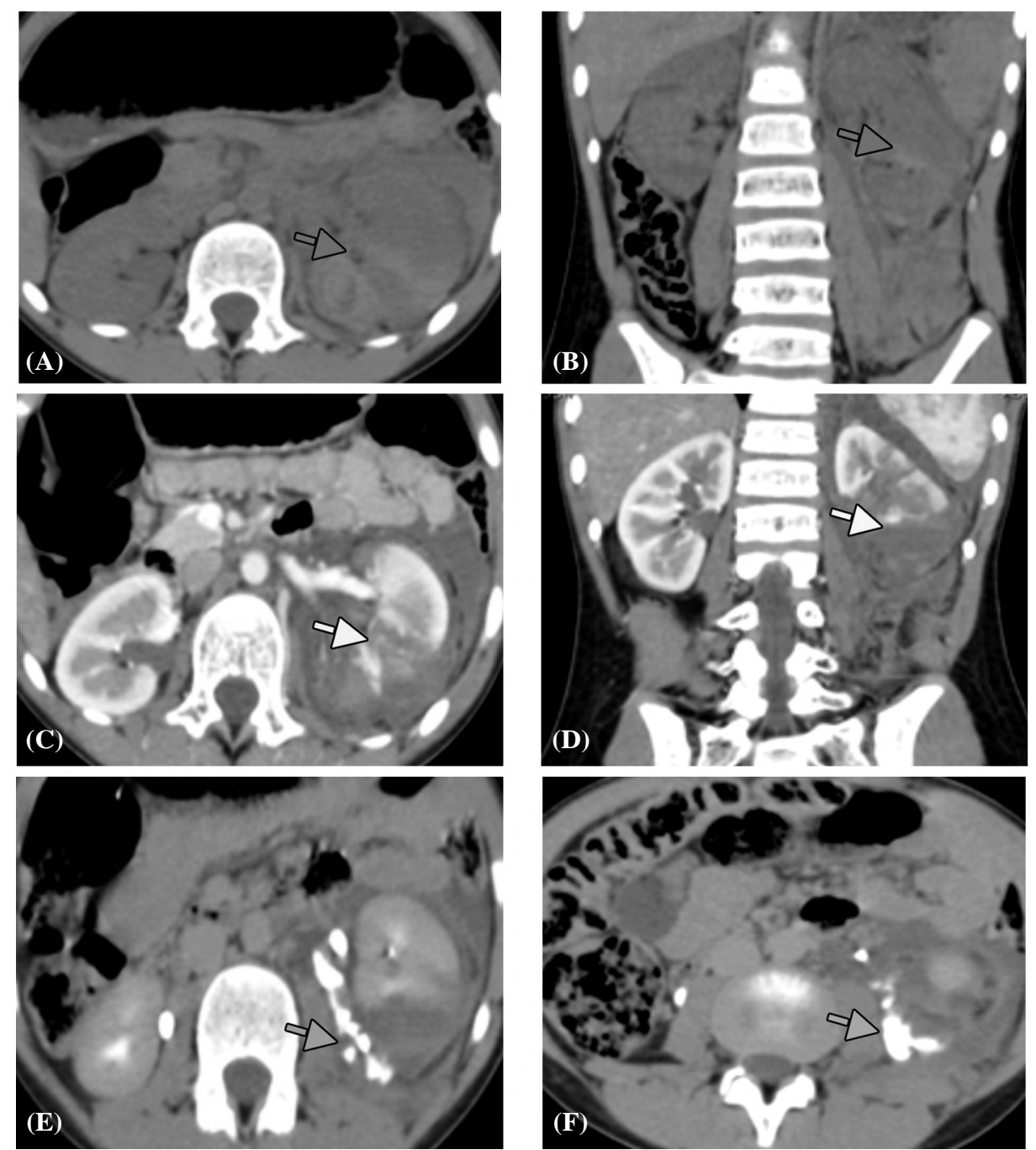

Fig. (1): A49-year-old male patient had history of blunt abdominal trauma since one day and presented with abdominal pain and haematuria.

Non contrast axial (A) and coronal MPR (B) CT of the abdomen and the pelvis: Revealed multiple linear hyperdense areas seen within left renal parenchyma (red arrow).

Post contrastaxial (C) and coronal MPR (D) CT of the abdomen and the pelvis: Revealed multiple deep parenchymal lacerations seen at middle and lower portion of the left kidney (yellow arrow) and left perinephric fluid collection.

Post contrast axial (E \& F) CT of the abdomen (excretory phase): Revealed extravasation of contrast enhanced urine (blue arrow) into the left perinephric collection (urinoma).

Impression: Shuttered left kidney (AAST grade V traumatic renal injury) with perinephric urinoma. 


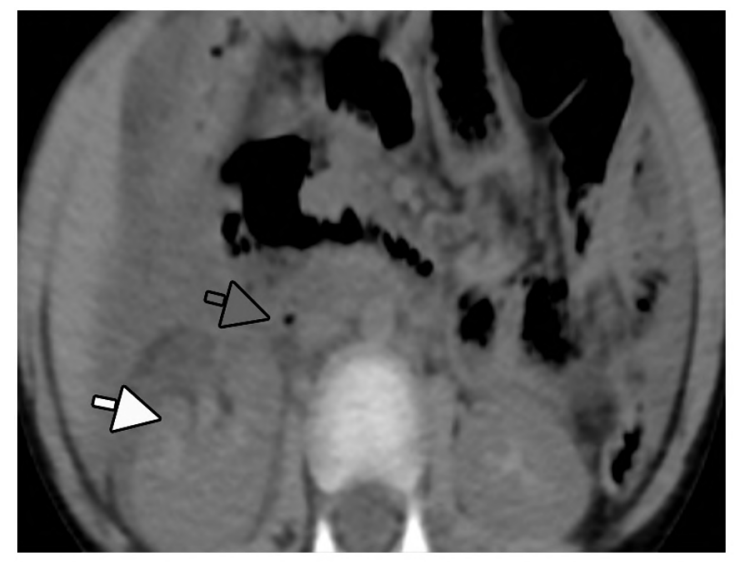

(A)

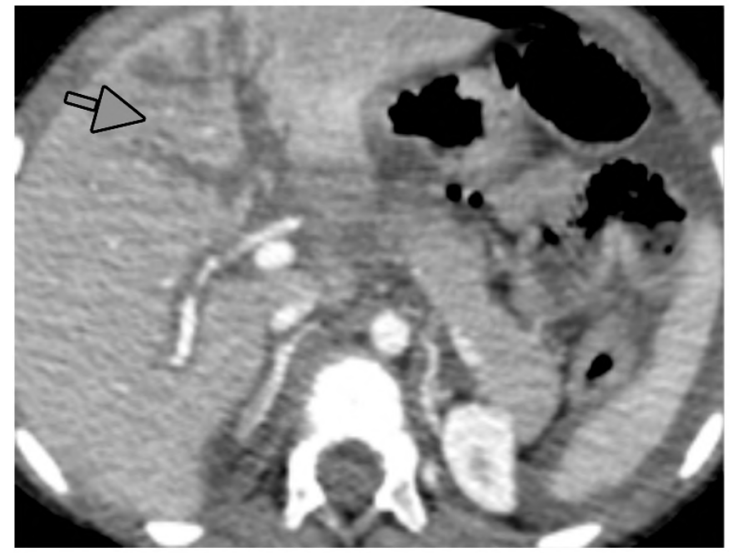

(C)

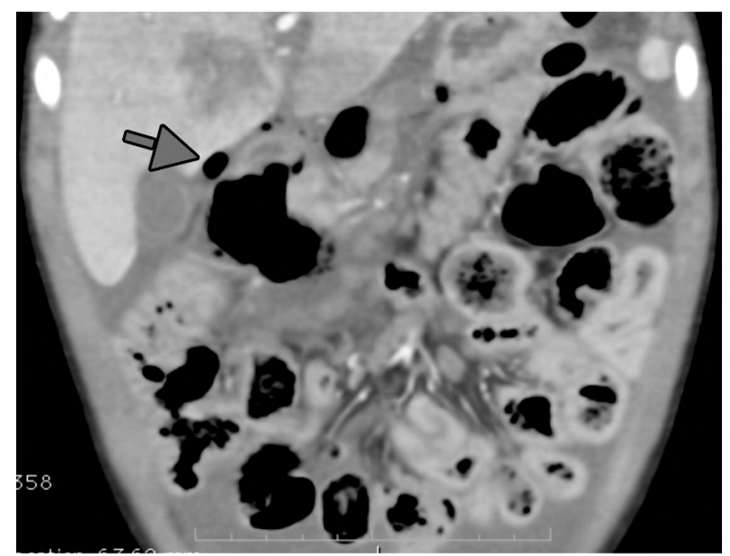

(E)

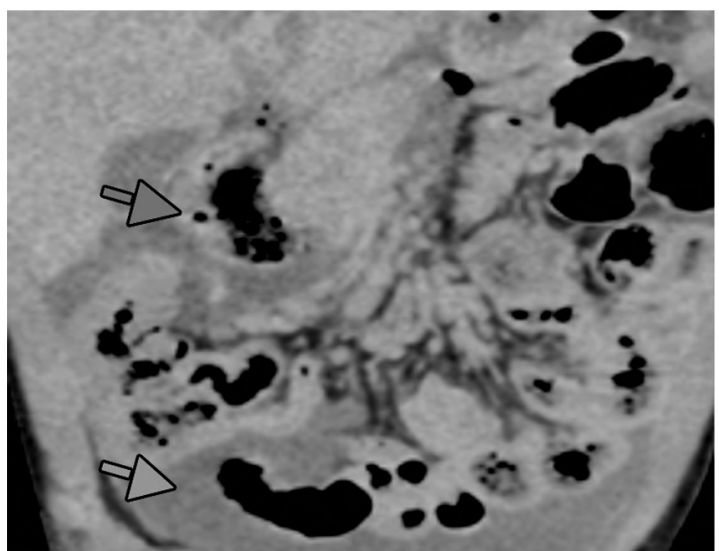

(B)

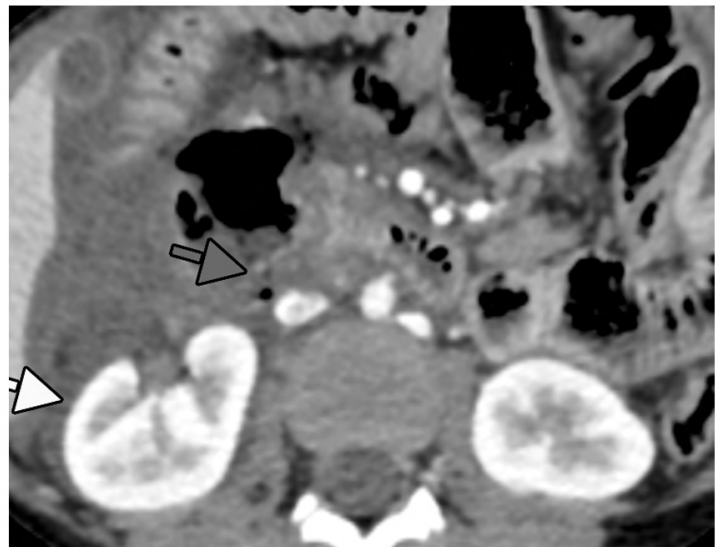

(D)

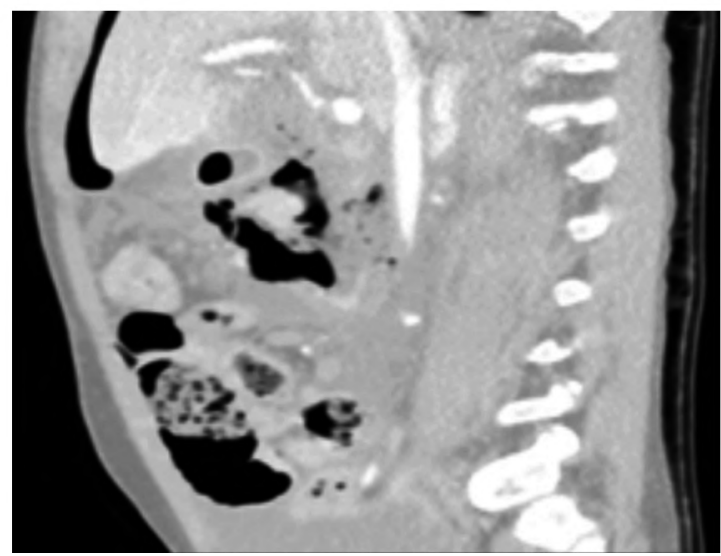

(F)

Fig. (2): A9-year-old male patient had history of blunt abdominal trauma (motor vehicle accident) since two days, he developed deterioration of vital signs with rapid decline of $\mathrm{HB}$ level reaching $8.5 \mathrm{gm} / \mathrm{dl}$.

Non contrast axial (A) and coronal MPR (B) CT of the abdomen and pelvis: Revealed extraluminal air seen adjacent to second part of the duodenum (red arrow), right perinephric fluid collection (yellow arrow) and intraperitoneal free fluid (green arrow).

Post contrast axial (C \& D), coronal MPR (E) and sagittal MPR (F) CT of the abdomen and pelvis: Revealed extraluminal air seen adjacent to second part of duodenum and at non dependent portion of the abdomen (red arrow), right perinephric hematoma, multiple deep laceration at the left hepatic lobe (blue arrow) and intraperitoneal free fluid.

Impression: Second part duodenal perforation associated with right perinephric hematoma, multiple deep laceration at the left hepatic lobe and intraperitoneal free fluid. 


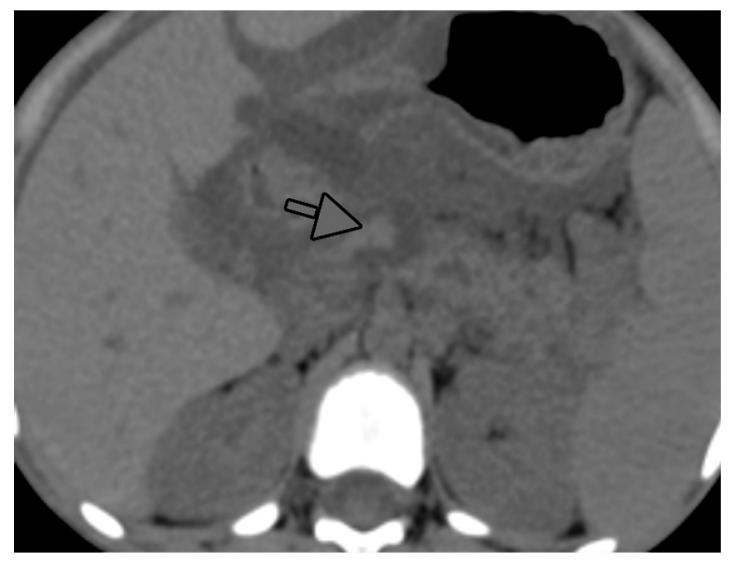

(A)

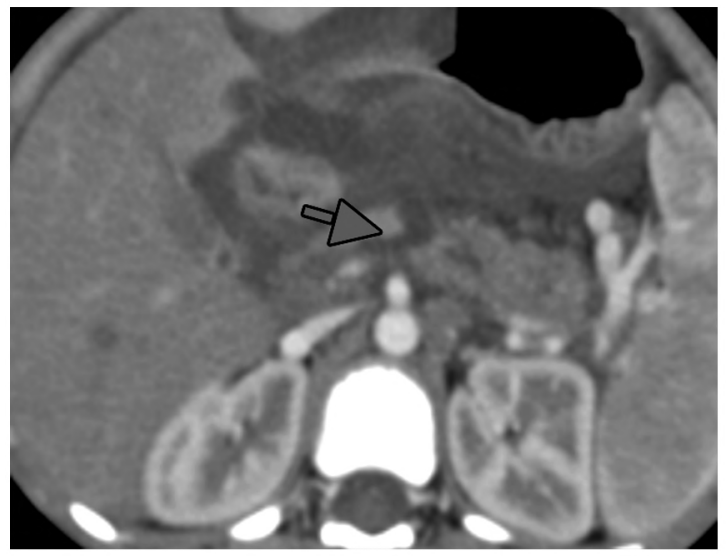

(C)

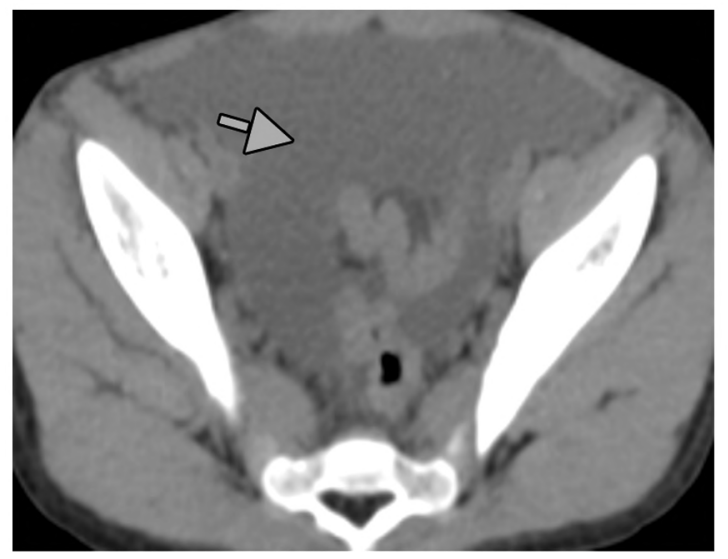

(E)

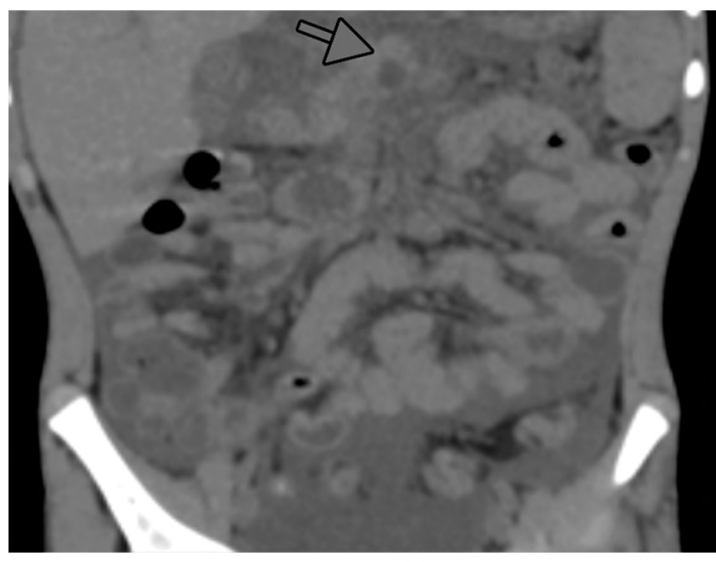

(B)

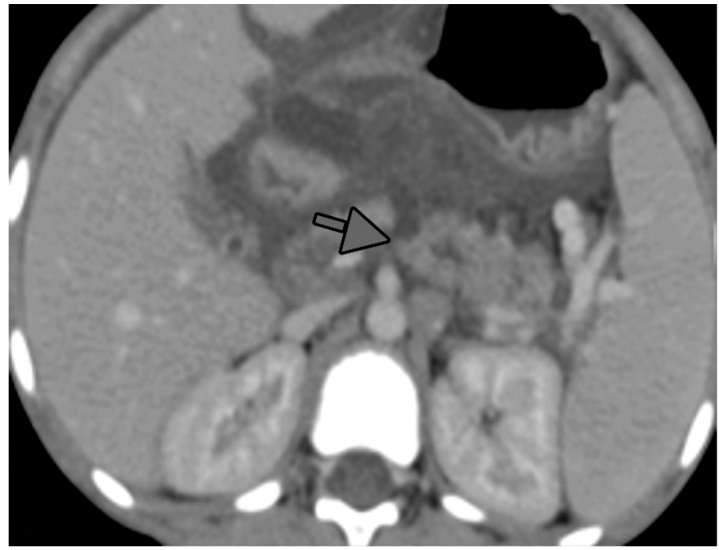

(D)

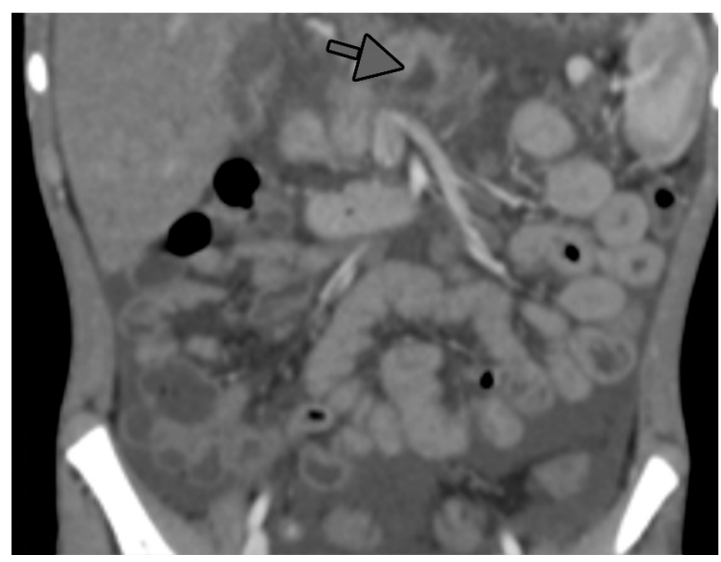

(F)

Fig. (3): A26-year-old male patient had history of penetrating trauma since 6 days, he developed deterioration of vital signs with rapid decline of HB level reaching $8 \mathrm{gm} / \mathrm{dl}$.

Non contrast axial (A) and coronal MPR (B) CT of the abdomen and pelvis: Revealed linear hypodense tear seen at the pancreatic body reaching to the main pancreatic duct (red arrow) and moderate intraperitoneal free fluid (blue arrow).

Post contrast axial (C, D \& E) and coronal MPR (F) CT of the abdomen and pelvis: Revealed linear area of diminished enhancement seen at the pancreatic body reaching the main pancreatic duct (red arrow) and moderate intraperitoneal free fluid (blue arrow).

Impression: Pancreatic body laceration reaching the main pancreatic duct (AAST grade III pancreatic traumatic injury). 


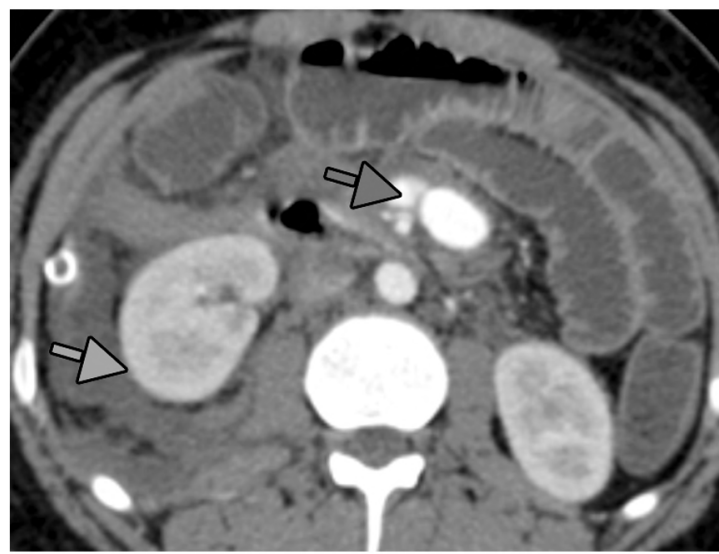

(A)

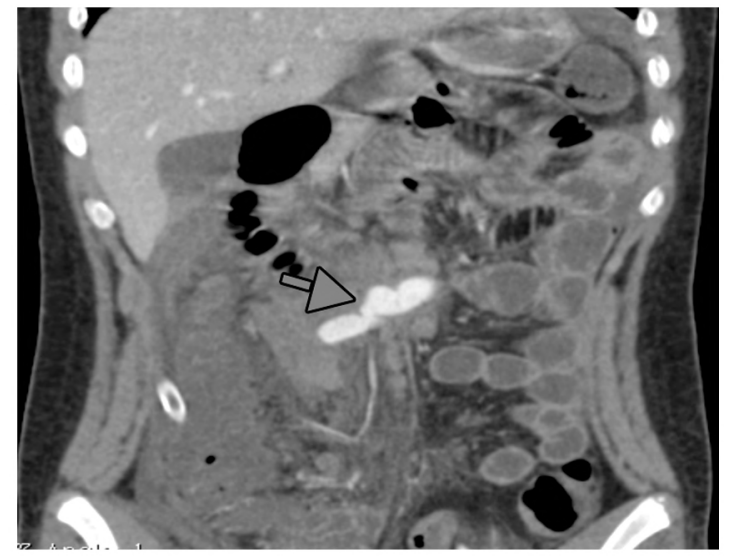

(B)

(C)

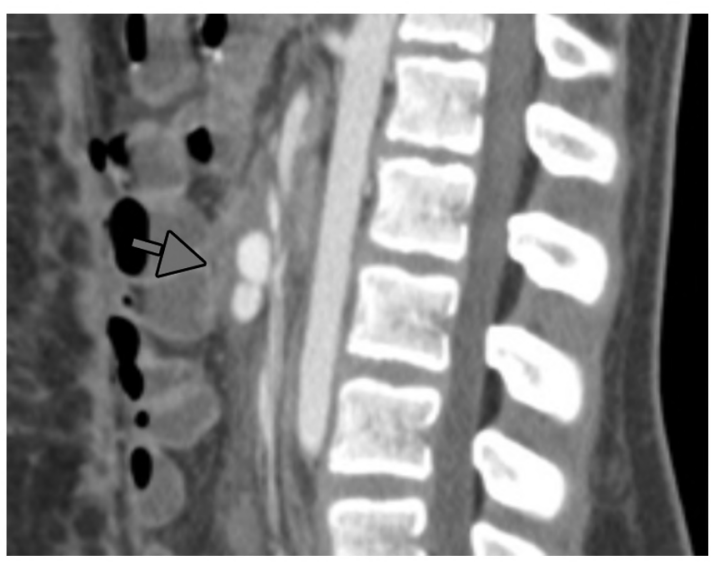

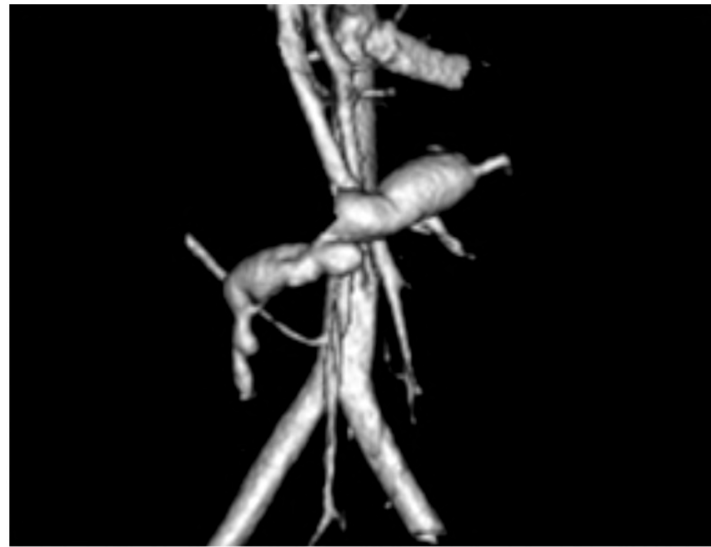

(D)

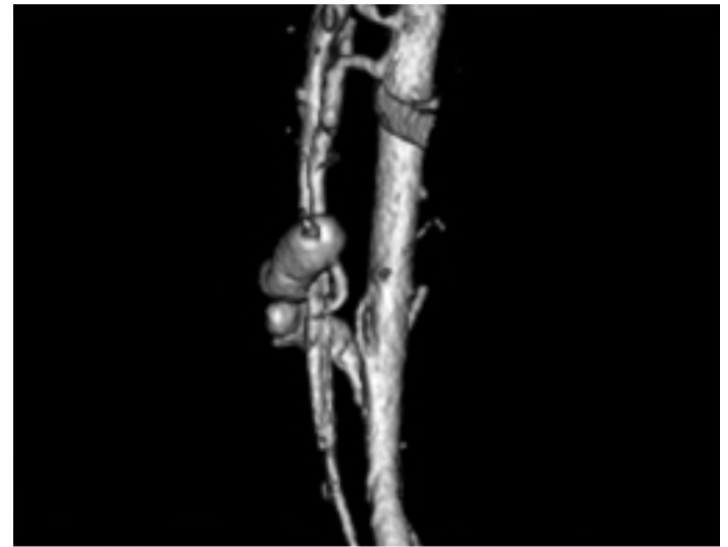

(E)

Fig. (4): A 41-year-old male patient had history of blunt abdominal trauma (battle) since one week and presented with abdominal pain.

Post contrast axial (A), coronal MPR (B) and sagittal MPR (C) CT of the abdomen and pelvis: Revealed arterial enhanced eccentric saccular aneurysmal dilatation seen at root of mesentery (red arrow) contiguous with the superior mesenteric artery and right sided perinepheric hematoma (blue arrow).

3D volume rendered CT angiography (D \& E): Revealed superior mesenteric artery pseudoaneurysm.

Impression: Superior mesenteric artery traumatic pseudoaneurysm and right perinephric hematoma. 

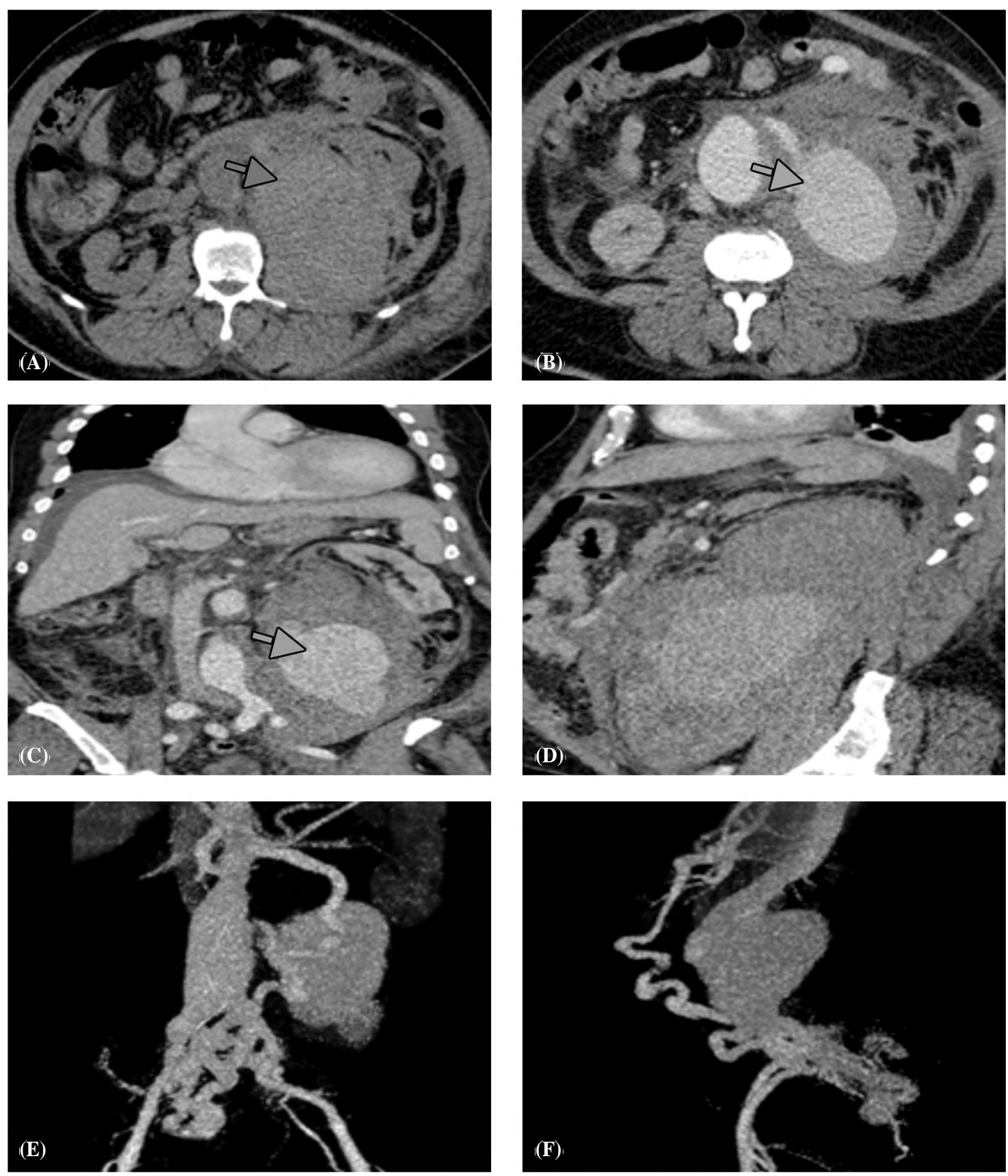

Fig. (5): A41-year-old male patient had history of blunt abdominal trauma (car accident) since 5 days, he developed deterioration of vital signs with rapid decline of $\mathrm{HB}$ level reaching $8 \mathrm{gm} / \mathrm{dl}$.

Non contrast axial (A) CT of the abdomen: Revealed large left paravertebral hyperdense collection (red arrow) seen displacing the left kidney anteriorly and laterally.

Post contrast axial (B), coronal MPR (C), sagittal MPR (D) CT of the abdomen, 3D volume rendered CT Angiography (E \& F): Revealed fusiform aneurysmal dilatation of infrarenal abdominal aorta with large eccentric saccular pseudoaneurysm seen left paravertebral region (blue arrow).

The impression: Abdominal aortic aneurysm traumatic rupture with pseudoaneurysm formation.

\section{Discussion}

Trauma is one of the leading causes of death worldwild and the abdomen is one of the most common sites of the body prone to injury, so the management of the patients requiring abdominal trauma needs speed, skill and efficiency [10].

In the present study, the male affection $(66.7 \%)$ was more than female affection (33.3\%), and there 
is no age group excluded from traumatic injury of the abdomen, but it was more common in 4 th decades, the adult were more common to abdominal trauma probably because of more exposure to day to day hazards.

These results were agreed with Awe et al., (2013) [11] who reported in their 5 years work that abdominal injury predominantly affects young male patients reaching the peak in twenty to fifty age groups and the incidence of male patients was $86.9 \%$ to female patients was $13.1 \%$, also agreed with Maske et al., (2016) [12] whose study was conducted on 50 patients with abdominal trauma; 36 of them $(72 \%)$ were male and 14 patients $(28 \%)$ were females and their ages distribution ranged from 11 years to 70 years with mean age (29.3) year.

Computed tomography is the imaging modality of choice in assessment of abdominal trauma in hemodynamically stable patients. With its high accuracy and sensitivity, it can determine the presence or absence of injury and its extent and thus helping the surgeon to take the proper decision in management and avoid unnecessary surgery. Because being less operator dependant and is not limited by the abdominal wall, subcutaneous emphysema, obesity, or intestinal distension, computed tomography outperforms ultrasound in detection of abdominal injuries [13,14]

Radiological evaluation by Multidetector Computed Tomography (MDCT) in this study revealed 30 patients $(50 \%)$ had retroperitoneal injuries and the other 30 patients (50\%) had other injuries rather than retroperitoneal injuries which were excluded from this study; 23 patients (38.33\%) had hepatic and splenic injuries and 7 patients $(11.67 \%)$ had only hemoperitoneum.

The percentage of retroperitoneal injuries occurrence among the patients with abdominal trauma in this study (50\%) does not cope with the study of El-Wakeel et al., (2015) [15] who found that retroperitoneal injuries occur with incidence $18 \%$ in the setting of abdominal trauma, and the liver was most common injured organ representing $40 \%$ followed by the spleen (30\%).

In the present study, the blunt abdominal trauma was the most common cause of retroperitoneal injuries representing $90 \%$ which is compatible with Dane et al., (2017) [16], they reported that 80-95\% of retroperitoneal injuries occur due to blunt trauma, whereas the remaining $5-20 \%$ were caused by penetrating traumas.
We observed that renal injuries were the most common injured retroperitoneal organ representing $50 \%$ of all patients followed by pancreatic injuries $8.33 \%$ then followed by adrenal and vascular injuries which representing 5\%. These results agreed with Ali et al., (2015) [17], they reported that the kidneys were the most common injured retroperitoneal organ on their study conducted on 110 patients with traumatic retroperitoneal injuries, the kidneys were injured in 34 patients (30.91\%).

In the current study, also we noticed that renal injuries associated with other injuries (66.67\%) more common than isolated renal injuries (33.33\%), adrenal injuries and pelvic fractures were the most common associated injuries.

Shaaban et al., (2016) [4] reported that renal injuries associated with other injuries more common than isolated renal injuries but liver lacerations were the most associated organ injuries found in 11 patients $(26.8 \%)$ and the spleen lacerations $(22 \%)$ were the second associated organ injuries found in 9 patients.

According to the American Association of Surgery of Trauma (AAST), renal trauma is divided into five categories (grades I-V) according to the severity and depth of injury and involvement of the vasculature or collecting system [18]

In the present study, Grade IV renal injury was the most common type of renal injury representing $33.33 \%$ of renal injuries which was agreed with Shaaban et al., (2016) [4] who reported that Grade I renal injury was diagnosed in $2.4 \%$ of patients, Grade II in $7.3 \%$, Grade III in $29.3 \%$, Grade IV in $53.7 \%$ and Grade $\mathrm{V}$ in $7.3 \%$, in the contrary, Alonso et al., (2009) [18] mentioned in his study that Grade I injury was the most common type of renal injury representing $75-85 \%$ of cases.

In the current study, adrenal injury was reported in $5 \%$ of the detected retroperitoneal injuries which agreed with Kevin et al., (2008) [1] who reported that adrenal injuries are not common as he found it in about $2 \%$ of abdominal trauma patients in his study.

In the current study, pancreatic injuries were reported in $16.67 \%$ of patients with abdominal trauma and Grade II injury was the most common type of pancreatic injury representing $40 \%$, which agreed with atusy done by Ali et al., (2015) [17], they reported that pancreatic injury Grade II was the most common injury representing $35.7 \%$ of pancreatic injuries. Fisher et al., (2011) [19] found that pancreatic trauma occurs in $14 \%$ of patients 
with abdominal trauma but Grade I pancreatic injury $(60 \%)$ representing the most common type of pancreatic injury.

In the current study the retroperitoneal bowel injury reported in two patient (6.67\%); one of them had duodenal perforation and the other had duodenal contusion, these results not agreed with Magu et al., (2012) [20] they reported on their study on 32 cases that duodenal injuries seen in 8 cases $(25 \%)$ and retroperitoneal bowel injuries found in 3 cases $(9.4 \%)$.

Ali (2016) [21] reported that retroperitoneal vascular injuries representing $13.3 \%$ of injuries in setting of abdominal and pelvic trauma, in the current study vascular injuries representing $5 \%$ of the retroperitoneal injuries.

We could reach the final diagnosis by detecting the site, the source and extent of retroperitoneal injury by MDCT in all cases, these results were agreed with Fang et al., (2006) [22] who reported that Multidetector CT has recently been shown to have a high degree of accuracy in the setting of abdominal trauma with a reported sensitivity and specificity approaching $100 \%$ in the initial assessment of trauma in 252 patients at one center.

\section{Conclusion:}

Multi-detector CT facilitates the recognition and evaluation of the retroperitoneal traumatic injuries and clarifies the grading and severity of these injuries with a great sensitivity and accuracy and thus appropriate management. Additionally, MDCT proved to be highly sensitive in detection of active hemorrhage which is a life threatening condition.

\section{References}

1- KEVIN P.D., CHRISTOPHER P.H., GAY S.B., et al.: Traumatic Retroperitoneal Injuries: Review of Multidetector CT Findings. Radiographics, 28 (6): 1571-90, 2008.

2- LEE E.S. and LEE J.M.: Imaging diagnosis of pancreatic cancer: A state-of-the-art. World J. Gastroenterol., 20 (24): 7864-77, 2014.

3- DAYAL M., GAMANAGATTI S. and KUMAR A.: Imaging in renal trauma. World Journal of Radiology, 5 (8): 275-84, 2013.

4- SHAABAN M.S., KHATTAB H.M.B., EL-SIRAFY M.N.I., et al.: Multi-detector CT assessment of traumatic renal lesions. Alexandria Journal of Medicine, 52 (2): 173-84, 2016.

5- SOTO J.A. and ANDERSON S.W.: Multidetector CT of blunt abdominal trauma. Radiology, 265 (3): 678-93, 2012.
6- MELAMUD K., LeBEDIS C.A. and SOTO J.A.: Imaging of pancreatic and duodenal trauma. Radiologic Clinics of North America, 53 (4): 757-71, 2015.

7- PATTERSON B.O., HOLT P.J., CLEANTHIS M., et al.: Imaging vascular trauma. British Journal of Surgery, 99 (4): 494-505, 2012.

8- GENOVESE E.A., FONIO P., FLORIDI C., et al.: Abdominal vascular emergencies: US and CT assessment. Critical Ultrasound Journal, 5 (1): 342-453, 2013.

9- STARNES B.W., LUNDGREN R.S., GUNN M., et al.: A new classification scheme for treating blunt aortic injury. Journal of Vascular Surgery, 55 (1): 47-54, 2012.

10-NERLI R.B., METGUD T., PATIL S., et al.: Severe renal injuries in children following blunt abdominal trauma: Selective management and outcome. Pediatric surgery international, 27 (11): 1213-16, 2011.

11- AWE J.A. and SOLIMAN A.M.: Abdominal trauma: A five year experience in a military hospital. Global Advanced Research Journal of Medicine and Medical Sciences, 2 (8): 177-83, 2013.

12- MASKE A.N. and DESHMUKH S.N.: Traumatic abdominal injuries: Our experience at rural tertiary care center. International Surgery Journal, 3 (2): 543-8, 2016.

13-AMERSTORFER E.E., HABERLIK A. and RICCABONA M.: Imaging assessment of renal injuries in children and adolescents: CT or ultrasound? Journal of pediatric surgery, 50 (3): 448-55, 2015.

14- ZAGO M.T., PEREIRA B.M.T. and FRAGA P.G.: Nonoperative management for patient with grade IV blunt hepatic trauma. World J. Emerg. Surg., 7: S8, 2012.

15- EL-WAKEEL A.M., HABIB R.M. and ALI A.N.: Role of CT in Evaluation of Blunt Abdominal Trauma. International Journal of Medical Imaging, 3: 89-93, 2015.

16-DANE B., BAXTER A.B. and BERNSTEIN M.P.: Imaging Genitourinary Trauma. Radiologic Clinics, 55 (2): 32135, 2017.

17-ALI A. and SHAHID M.R.: Frequency of Mode and Grade of Retroperitoneal Organ Injuries following Blunt $\mathrm{Ab}$ dominal Trauma. Pakistan Journal of Medical and Health Sciences, 9: 134-141, 2015.

18- ALONSO R.C., NACENTA S.B., MARTINEZ P.D., et al.: Kidney in danger: CT findings of blunt and penetrating renal trauma. Radiographics, 29 (7): 2033-53, 2009.

19- FISHER M. and BRASEL K.: Evolving management of pancreatic injury. Current Opinion in Critical Care, 17 (6): 613-7, 2011.

20- MAGU S., AGARWAL S. and GILL R.S.: Multi detector computed tomography in the diagnosis of bowel injury. Indian Journal of Surgery, 74 (6): 445-50, 2012.

21- ALI H.I.: Role of multislice computed tomography in assessment of non-solid organ injury in patients with blunt abdominal trauma. The Egyptian Journal of Radiology and Nuclear Medicine, 47 (3): 749-56, 2016.

22- FANG J.F., WONG Y.C., LIN B.C., et al.: Usefulness of multidetector computed tomography for the initial assessment of blunt abdominal trauma patients. World Journal of Surgery, 30 (2): 176-82, 2006. 


\section{دور الآشعة المقطعية متعددة الكواشف

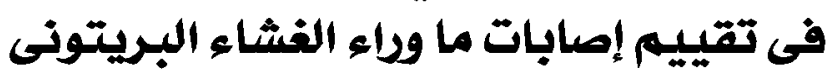

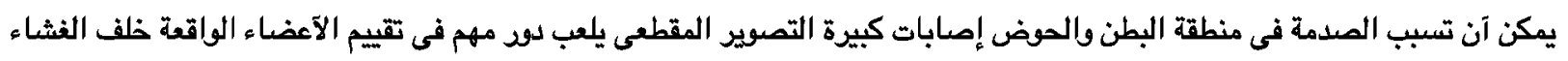

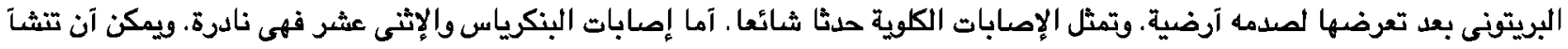

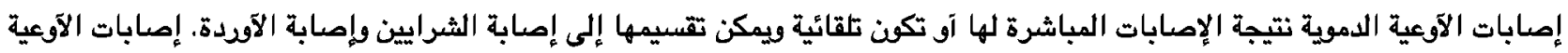

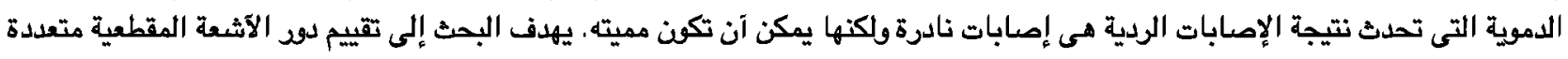
الكواشف فى تقييم إصابات ما وراء الغشاء البريتونى. 